\title{
DIVERGÊNCIA GENÉTICA ENTRE GENÓTIPOS DE FEIJOEIRO A PARTIR DE TÉCNICAS MULTIVARIADAS
}

\author{
GENETIC DIVERGENCE AMONG GENOTYPES OF COMMON BEAN \\ TROUGHT OF MULTIVARIATES TECHNIQUES
}

\author{
Cristina de Fátima Machado ${ }^{1}$ Glauber Henrique de Sousa Nunes ${ }^{2}$ \\ Daniel Furtado Ferreira ${ }^{3}$ João Bosco dos Santos ${ }^{4}$
}

\section{RESUMO}

Foram avaliadas as técnicas multivariadas para se estimar a divergência genética entre genótipos de feijoeiro, visando a obtenção de populações com ampla variabilidade genética. Utilizaram-se as seguintes técnicas de análise multivariada: distância Euclidiana média, a partir dos dados padronizados $\left(d_{e}\right)$; distância Euclidiana média, obtida com os escores das três primeiras variáveis canônicas $\left(d_{v c}\right)$; distância Euclidiana média, a partir dos escores dos três primeiros componentes principais $\left(d_{c p}\right)$; distância Euclidiana média usando as três primeiras cargas fatoriais $\left(d_{f t}\right)$, e distância generalizada de Mahalanobis $\left(D^{2}\right)$. Foram utilizados doze genótipos de feijoeiro (Aporé, H-4-7, PF-9029975, CI-128, Carioca MG, CI21, Carioca 300V, Ouro Negro, A-285-Rudá, ESAL 693, Pérola e IAC Carioca Aruã) avaliados em quatro épocas (inverno/97, águas/97/98, seca/98 e inverno/98) por meio de dez. características agromorfológicas. $O$ delineamento utilizado foi blocos completos casualizados com três repetições. As técnicas multivariadas foram concordantes para identificar os genótipos geneticamente mais divergentes. Os genótipos, ESAL 693 e Ouro Negro diferiram entre si e entre os demais. O PF-9029975 e Carioca MG foram similares e diferiram das demais de acordo com as distâncias Euclidianas médias. Salienta-se também a divergência da cultivar Aporé em relação à Pérola, embora esta cultivar seja uma linhagem selecionada dentro da Aporé. Assim, todos esses genótipos geneticamente mais divergentes são promissoros para serem cruzados e fornecerem populações com maior segregação em vários caracteres agronômicos, especialmente o ESAL 693 com os demais que possuem grãos tipo carioca. Foi também constatada razoável concordância na identificação dos caracteres que menos contribuíram para a diversidade genética.
Palavras-chave:

caracteres agromorfológicos, distância euclidiana média, componentes principais, variáveis canônicas, fatorial, distância generalizada de Mahalanobis $\left(D^{2}\right)$.

SUMMARY

Multivariate techniques were used to evaluate the genetic divergence among genotypes of common bean, aiming to identify segregating populations with large genetic variability. The multivariate techniques used were: the average Euclidean distance based on standardized variables $\left(d_{e}\right)$, on scores of the three first canonical variables $\left(d_{v c}\right)$, on scores of the first three principal components $\left(d_{c p}\right)$, on the first three factor loads $\left(d_{f t}\right)$, and the Mahalanobis generalized distance $\left(D^{2}\right)$. Twelve common bean genotypes were used (Aporé, H-4-7, PF-9029975, CI-128, Carioca MG, CI-21, Carioca 300V, Ouro Negro, A-285 Rudá, ESAL 693, Pérola e IAC Carioca Aruã), and evaluated in four seasons (Jul-Nov/97, Nov/97-Feb/98, Feb-Jun/98 and JulNov/98), based on ten morpho-agronomic traits. A randomized block design with three replications was utilized. The multivariate techniques showed similar results mainly for identifing the more divergent genotypes. Among them, ESAL 693 and Ouro Negro were genetically different between themselves and among the other genotypes. PF-9029975 and Carioca MG were genetically similar, although different of other genotypes according to average Euclidean procedures. It should be pointed out that Aporé genotype was divergent of Pérola genotype, although the last one is a selected line in the Aporé. Therefore, all of those genetically divergent genotypes are promissing to cross for obtaining higher segregating populations, specially ESAL 693 with the others that have a carioca grain type. A resonable correspondence was found in the identification of traits with smallest contribution to genetic diversity.

\footnotetext{
${ }^{1}$ Engenheiro Agrônomo, Doutor em Genética e Melhoramento, Departamento de Biologia, Universidade Federal de Viçosa. E-mail: crisagornoma@yahoo.com.br. Autor para correspondência.

${ }^{2}$ Engenheiro Agrônomo, Genética e Melhoramento de Plantas, Departamento de Biologia da Universidade Federal de Lavras (UFLA).

${ }^{3}$ Engenheiro Agrônomo, Doutor, Departamento de Ciências Exatas, Bolsista CNPq, UFLA. E-mail: daniel@ufla.br.

${ }^{4}$ Engenheiro Agrônomo, Doutor, Departamento de Biologia, UFLA. E-mail: jbsantos@ufla.br.
} 
Key words:morpho-agronomic traits, Euclidean distance, canonical variables, principal components, factorial, Mahalanobis $\left(D^{2}\right)$ generalized distance.

\section{INTRODUÇÃO}

O melhoramento do feijoeiro no Brasil se baseia, principalmente, na hibridação de cultivares ou linhagens para gerar populações segregantes, nas quais se procede a seleção de linhagens superiores. Nesse contexto, os estudos sobre divergência genética podem ser de grande importância por fornecerem estimativas para a identificação de genitores que, quando cruzados, aumentem as chances de seleção de genótipos superiores nas gerações segregantes (CRUZ et al., 1994).

Vários métodos multivariados podem ser aplicados no estudo da divergência genética. Dentre eles, destacam-se a análise de componentes principais, de variáveis canônicas e os métodos de agrupamentos. A escolha do método mais adequado, tem sido determinada de acordo com os objetivos do pesquisador, pela facilidade da análise e pela forma como os dados foram obtidos (MIRANDA et al., 1988; CRUZ, 1990; CRUZ et al., 1994).

A viabilidade da utilização de técnicas multivariadas no estudo da divergência genética como auxílio na seleção de genitores para cruzamentos tem sido aplicada em várias culturas. Em feijão (ADAMS, 1977; GHADERI et al., 1984, OLIVEIRA, 1989), em milho (CRUZ et al., 1994), em arroz (JULQUIFAR et al., 1985), em pimenta (CUARTERO et al. 1983), em milheto (JAIN et al.1981a), em grão-de-bico (JAIN $\boldsymbol{e t}$ $\boldsymbol{a l} ., 1981 b$ ), em azevém (FARIAS $\boldsymbol{e t} \boldsymbol{a l} ., 1983$ ), em feijão-vagem (MALUF $\boldsymbol{e t}$ al., 1983) em pimentão (MIRANDA et al.,1988), em soja MIRANDA (1998). Com base nessas considerações, os objetivos deste trabalho foram: avaliar a divergência genética entre cultivares/linhagens de feijão; discriminar os caracteres mais importantes na avaliação da divergência genética pelas estatísticas multivariadas, e determinar o grau de concordância das estimativas da divergência genética.

\section{MATERIAL E MÉTODOS}

Foram utilizados doze genótipos de feijoeiro (Aporé, H-4-7, PF-9029975, CI-128, Carioca MG, CI-21, Carioca 300V, Ouro Negro, A285-Rudá, ESAL 693, Pérola e IAC Carioca Aruã) avaliados em quatro épocas (inverno/97, águas/97/98, seca/98 e inverno/98) por meio de dez características agromorfológicas. O delineamento utilizado foi blocos completos casualizados com três repetições. Esses genótipos foram escolhidos por apresentarem características agronômicas favoráveis, como porte, hábito de crescimento e produtividade, sendo estas características importantes para o melhoramento (MACHADO et al., 2000, MACHADO, et al., 2002). A densidade de semeadura empregada foi estabelecida conforme os objetivos dos programas de melhoramento para à Região Sul de Minas Gerais, sendo assim, cada parcela consistiu de duas linhas de 3 metros de comprimento, com espaçamento de $0,50 \mathrm{~m}$ entre as linhas e 15 sementes por metro linear (VIEIRA, 1991, ABREU, 1999, VIEIRA et al., 2000).

A avaliação dos genótipos foi feita por meio dos seguintes caracteres agronômicos: 1 - FL: número de dias para o florescimento (anotada quando $50 \%$ das plantas da parcela já apresentavam pelo menos um flor aberta), 2 - NLP: número médio de legumes por planta, 3 - NI: número médio de internódios da haste principal, 4 - PS: peso médio de 100 sementes, em g, 5 - PD: produção de grãos em $\mathrm{kg} / \mathrm{ha}, 6$ - NSL: número médio de sementes por legume, 7 - IL: inserção média do primeiro legume até o colo da planta, em $\mathrm{cm}, 8$ - NR: número médio de ramos por planta, 9 - HP: comprimento médio da haste principal, do colo até a inserção da última folha, em cm, 10 - PR: porte das plantas, através de escala de notas, segundo OLIVEIRA (1996). Na avaliação dos genótipos, foram utilizadas todas as plantas da parcela para se obter PD, FL e PR. Os demais caracteres foram avaliados a partir de uma amostra de dez plantas por parcela.

A análise de variância multivariada, para a avaliação da variabilidade genética total existente entre os genótipos foi feita a partir dos dados provenientes da análise conjunta de variância das quatro épocas para cada caráter, na qual se consideraram os efeitos de genótipos e épocas como fixos (MACHADO et al., 2000). A partir da análise de variância multivariada, obtiveram-se as matrizes de soma de quadrados e produtos residual, a matriz de soma de quadrados e produtos entre genótipos. As distâncias generalizadas de Mahalanobis e as distâncias Euclidianas médias, obtidas a partir dos dados padronizados, foram utilizadas como medidas de dissimilaridades. As medidas de dissimilaridade entre cada par de genótipos foram obtidas a partir das cinco técnicas multivariadas: a) distância Euclidiana média, a partir dos dados padronizados $\left.\left(\mathrm{d}_{\mathrm{e}}\right), \mathrm{b}\right)$ distância Euclidiana média, com os escores das três primeiras variáveis canônicas $\left(\mathrm{d}_{\mathrm{vc}}\right)$, c) distância Euclidiana média, a partir dos escores dos três primeiros componentes principais $\left(\mathrm{d}_{\mathrm{cp}}\right)$, d) distância Euclidiana média, obtida, com o uso das três primeiras cargas fatoriais $\left(\mathrm{d}_{\mathrm{ft}}\right)$ e, e) distância generalizada de Mahalanobis $\left(\mathrm{D}^{2}\right)$. 
As médias de cada uma das variáveis foram expressas em unidades de desvio-padrão e utilizadas na obtenção dos dez componentes principais, utilizando-se os recursos computacionais do Programa GENES (CRUZ, 1997). Para as estimativas dos autovalores e variâncias foram consideradas os três primeiros componentes principais e as três primeiras variáveis canônicas, que acumulam uma explicação da variância superior a $79 \%$ e $99 \%$ respectivamente, que é considerada satisfatória para dados biológicos (ISEBRANDS \& CROW, 1975, JOHNSON \& WICHERN, 1992, OLIVEIRA, 1989, FERREIRA, 1993, CRUZ \& REGAZZI, 1997). A divergência entre os genótipos foi quantificada pelas medidas de dissimilaridade, segundo JOHNSON \& WICHERN (1992) e CRUZ \& REGAZZI (1997). Para formação dos grupos, foi utilizado o método hierárquico aglomerativo da média entre pares não ponderados (UPGMA) conforme descrito por DIAS (1998).

O grau de concordância das estimativas da divergência genética, quantificada pelas cinco estatísticas multivariadas, descritas anteriormente, foi avaliado pelo coeficiente de correlação de Spearman. Testou-se a hipótese de que a matriz de correlações residuais não difere da matriz identidade (Ho: $\mathrm{R}=\mathrm{I}$ ), conforme procedimento sugerido por GODOI (1985). Avaliou-se, também, a concordância das técnicas dos componentes principais e das variáveis canônicas na discriminação da importância de variáveis no estudo da divergência genética, segundo MARDIA $\boldsymbol{e t}$ al. (1979). Esse mesmo critério foi adotado para as variáveis canônicas, diferindo quanto aos coeficientes de ponderação das variáveis originais, os quais foram previamente multiplicados pelo desvio-padrão residual, conforme sugerido por TATSUOKA (1971).

\section{RESULTADOS E DISCUSSÃO}

Verifica-se que houve concordância parcial entre os resultados pela aplicação dos cinco métodos empregados. Entretanto, deve-se ressaltar que os genótipos PF-9029975 e o ESAL 693 foram os mais divergentes para a $\mathrm{d}_{\mathrm{e}}$; já os genótipos Ouro Negro e Pérola foram os mais divergentes para as $\mathrm{d}_{\mathrm{vc}}$, os genótipos CI-21 e ESAL 693 os mais divergentes para os $\mathrm{d}_{\mathrm{cp}}$, os genótipos Carioca MG e Pérola foram os mais divergentes para as $\mathrm{d}_{\mathrm{ft}} \mathrm{e}$ os genótipos PF-9029975 e ESAL 693 foram os mais divergentes para a $\mathrm{D}^{2}$ (Tabela 1 ). Vale ressaltar que, de maneira geral, o genótipo ESAL 693 foi considerado o mais divergente pelas cinco técnicas aplicadas. Analisando ainda a tabela 1, percebe-se que este genótipo apresentou, na maioria das vezes, as maiores dissimilaridades, sendo que de onze combinações, oito apresentaram valores acima de 1,800 para uma das quatro técnicas utilizadas. Já os valores para a $\mathrm{D}^{2}$ variaram de 2,770 (Aporé e Carioca 300V) até 96,350 (ESAL 693 e PF9029975). Entre as distâncias de cada par de genótipos, oito também apresentaram valores acima de 60,000 sendo que seis dessas combinações possuem o genótipo ESAL 693 (MACHADO et al., 2000).

Houve concordância entre as cinco técnicas empregadas para a discriminação dos genótipos mais divergentes. Estes resultados podem ser visualizados pelos coeficientes de correlação de Spearman (Tabela 2), todas foram positivas, significativas e maiores ou igual a 0,630. Estes resultados concordam em parte com os obtidos em milho por CRUZ et al. (1994) e Ferreira, (1993), que encontraram grande concordância pela aplicação da distância de Mahalanobis, distância Euclidiana média, componentes principais e variáveis canônicas, evidenciado pelo coeficiente de correlação de Spearman superior (0.93 e 0.58), respectivamente.

A correlação entre as estimativas de $\mathrm{D}^{2}$ e $d_{e}(r=0,701 * *)$, e $d_{c p}$ e $d_{v c}(r=0,733 * *)$, e $d_{e}$ e $d_{c p}$ $\left(\mathrm{r}=0,883^{* *}\right), \mathrm{d}_{\mathrm{ft}}$ e $\mathrm{d}_{\mathrm{e}}\left(\mathrm{r}=0,874^{* *}\right)$, e $\mathrm{d}_{\mathrm{vc}}$ e $\mathrm{D}^{2}$ $(\mathrm{r}=0,847 * *) \quad$ apresentaram magnitudes consideráveis, uma vez que a matriz de correlações residuais é estatisticamente igual à matriz identidade (Tabela 2). Tal fato tem reflexos em estudos de melhoramento, pois os resultados obtidos em estudos sobre divergência, mesmo onde não se dispõe de meios para quantificar a variação e a co-variação residuais, são válidos e úteis, principalmente quando essas técnicas multivariadas são utilizadas. Entre a média dos genótipos, destacaram-se as correlações entre os seguintes caracteres: NI e HP, NI e NR, NLP e NR, NR e HP e NR, IL e HP, HP e PR, NLP e NI, NLP e PD, e PD e NSL. Apesar da significância dessas correlações, estes caracteres não apresentaram contribuições individuais significativas para a variabilidade total, como pode ser observado pelos três primeiros componentes principais e variáveis canônicas (autovetores) de maior importância (Tabelas 3 e 4). Vale ressaltar que NI e HP, e NI e NR apresentaram os maiores valores de correlações $(0,707$ e 0,580) respectivamente, sendo portanto, importantes. Uma das explicações para a variação entre os resultados apresentados pelas cinco estatística multivariadas foi devido a baixa magnitude das correlações residuais apresentada para a maioria dos caracteres avaliados, e também pelo fato de que a matriz de correlações residuais é estatisticamente igual a matriz identidade. 
Tabela 1 - Medidas de dissimilaridade entre pares de cultivares/linhagens de feijão obtidas pela distância euclidiana média a partir de dados padronizados ( $\mathrm{d}_{\mathrm{e}}$ ), dos escores das três primeiras variáveis canônicas $\left(\mathrm{d}_{\mathrm{vc}}\right)$, dos três primeiros componentes principais $\left(\mathrm{d}_{\mathrm{cp}}\right)$, dos escores das três primeiras cargas fatoriais $\left(\mathrm{d}_{\mathrm{ft}}\right)$, e a partir da distância generalizada de Mahalanobis $\left(\mathrm{D}^{2}\right)$, estimados a partir da avaliação média de 10 caracteres agromorfológicos em quatro épocas (inverno/97, águas/97/98, seca/98 e inverno/98).

\begin{tabular}{|c|c|c|c|c|c|c|c|c|c|c|c|c|c|}
\hline Genótipos & $\begin{array}{c}\text { Medidas de } \\
\text { dissimilaridades }\end{array}$ & Aporé & $\mathrm{H}-4-7$ & $\begin{array}{c}\text { PF- } \\
9029975\end{array}$ & CI-128 & $\begin{array}{c}\text { Carioca } \\
\text { MG }\end{array}$ & CI-21 & $\begin{array}{l}\text { Carioca } \\
300 \mathrm{~V}\end{array}$ & $\begin{array}{c}\text { Ouro } \\
\text { Negro }\end{array}$ & $\begin{array}{l}\text { A-285 } \\
\text { Rudá }\end{array}$ & $\begin{array}{l}\text { ESAL- } \\
693\end{array}$ & $\begin{array}{l}\text { Pérola } \\
\text { IAC }\end{array}$ & $\begin{array}{l}\text { Carioca } \\
\text { Aruã }\end{array}$ \\
\hline \multirow[t]{5}{*}{ 1-Aporé } & $\left(d_{e}\right)$ & - & 1,421 & 1,618 & 0,686 & 1,775 & 1,288 & 0,523 & 1,417 & 1,106 & 1,896 & 0,582 & 1,369 \\
\hline & $\left(d_{y c}\right)$ & - & 1,099 & 1,837 & 0,591 & 1,508 & 0,791 & 0,333 & 1,636 & 1,071 & 1,553 & 1,155 & 0,724 \\
\hline & $\left(\mathrm{d}_{\mathrm{cp}}\right)$ & - & 1,466 & 1,438 & 0,801 & 1,521 & 1,252 & 0,608 & 1,162 & 1,203 & 1,609 & 0,775 & 1,491 \\
\hline & $\left(\mathrm{d}_{\mathrm{ft}}\right)$ & - & 1,557 & 1,882 & 0,776 & 2,280 & 1,282 & 0,575 & 1,464 & 1,457 & 1,868 & 0,345 & 1,459 \\
\hline & $\left(\mathrm{D}^{2}\right)$ & - & 19,430 & 30,960 & 5,400 & 21,660 & 10,990 & 2,770 & 30,110 & 16,380 & 47,450 & 7,250 & 14,110 \\
\hline \multirow[t]{5}{*}{ 2-H-4-7 } & $\left(\mathrm{d}_{\mathrm{e}}\right)$ & & - & 1,589 & 1,368 & 1,249 & 0,620 & 0,987 & 1,057 & 0,825 & 1,635 & 1,876 & 0,357 \\
\hline & $\left(d_{\mathrm{vc}}\right)$ & & - & 1,818 & 1,528 & 1,448 & 0,374 & 0,772 & 1,469 & 1,394 & 1,999 & 2,164 & 0,915 \\
\hline & $\left(d_{\mathrm{cp}}\right)$ & & - & 1,559 & 1,558 & 1,337 & 1,073 & 1,171 & 1,589 & 1,310 & 1,907 & 1,786 & 1,087 \\
\hline & $\left(\mathrm{d}_{\mathrm{ft}}\right)$ & & - & 1,208 & 26,190 & 1,222 & 0,367 & 0,986 & 1,286 & 0,511 & 1,895 & 1,895 & 0,363 \\
\hline & $\left(\mathrm{D}^{2}\right)$ & & - & 19,580 & 1,575 & 13,220 & 7,540 & 12,810 & 43,670 & 19,130 & 71,480 & 41,640 & 12,260 \\
\hline \multicolumn{14}{|l|}{ 3-PF- } \\
\hline \multirow[t]{5}{*}{9029975} & $\left(d_{e}\right)$ & & & - & 1,683 & 0,579 & 1,963 & 1,683 & 1,354 & 1,025 & 2,196 & 1,545 & 1,771 \\
\hline & $\left(\mathrm{d}_{\mathrm{vc}}\right)$ & & & - & 1,462 & 0,385 & 1,885 & 1,734 & 1,774 & 0,902 & 2,001 & 1,935 & 1,223 \\
\hline & $\left(\mathrm{d}_{\mathrm{cp}}\right)$ & & & - & 1,510 & 0,627 & 1,760 & 1,464 & 1,457 & 1,051 & 1,956 & 1,369 & 1,660 \\
\hline & $\left(\mathrm{d}_{\mathrm{ft}}\right)$ & & & - & 39,140 & 0,524 & 1,390 & 1,496 & 0,809 & 0,828 & 1,423 & 2,110 & 1,505 \\
\hline & $\left(\mathrm{D}^{2}\right)$ & & & - & - & 2,900 & 19,650 & 21,590 & 62,180 & 7,460 & 96,350 & 44,010 & 13,170 \\
\hline \multirow[t]{5}{*}{ 4-CI-128 } & $\left(d_{e}\right)$ & & & & - & 1,630 & 1,463 & 0,847 & 0,906 & 1,307 & 1,259 & 0,911 & 1,468 \\
\hline & $\left(\mathrm{d}_{\mathrm{vc}}\right)$ & & & & - & 1,416 & 1,300 & 0,835 & 1,548 & 1,036 & 1,177 & 0,793 & 0,937 \\
\hline & $\left(\mathrm{d}_{\mathrm{cp}}\right)$ & & & & - & 1,524 & 1,596 & 0,991 & 1,251 & 1,461 & 1,001 & 0,740 & 1,608 \\
\hline & $\left(\mathrm{d}_{\mathrm{ft}}\right)$ & & & & - & 1,936 & 1,445 & 0,881 & 0,856 & 1,412 & 1,110 & 0,886 & 1,635 \\
\hline & $\left(\mathrm{D}^{2}\right)$ & & & & - & 29,580 & $\begin{array}{r}1,4+30 \\
18,280\end{array}$ & $\begin{array}{l}0,001 \\
7,630\end{array}$ & $\begin{array}{r}0,000 \\
29,440\end{array}$ & $\begin{array}{r}15,180 \\
25,180\end{array}$ & 27,640 & 8,040 & 21,840 \\
\hline \multicolumn{14}{|l|}{ 5-Carioca } \\
\hline \multirow[t]{5}{*}{ MG } & $\left(\mathrm{d}_{\mathrm{e}}\right)$ & & & & & - & 1,751 & 1,677 & 1,114 & 0,970 & 1,957 & 1,871 & 1,502 \\
\hline & $\left(\mathrm{d}_{\mathrm{vc}}\right)$ & & & & & - & 1,517 & 1,378 & 1,483 & 0,704 & 1,770 & 1,793 & 0,897 \\
\hline & $\left(d_{\mathrm{cp}}\right)$ & & & & & - & 1,550 & 1,454 & 1,375 & 0,935 & 1,862 & 1,593 & 1,386 \\
\hline & $\left(\begin{array}{l}\left.\mathrm{c}_{\mathrm{cp}}\right) \\
\left.\mathrm{d}_{\mathrm{ft}}\right)\end{array}\right.$ & & & & & - & 1,505 & 1,817 & 1,180 & 0,985 & 1,712 & 2,542 & 1,557 \\
\hline & $\left(\mathrm{D}^{2}\right)$ & & & & & - & 12,080 & 15,060 & 47,750 & 5,210 & 80,140 & 36,030 & 8,020 \\
\hline \multirow[t]{5}{*}{ 6-CI-21 } & $\left(\mathrm{d}_{\mathrm{e}}\right)$ & & & & & & - & 0,768 & 1,508 & 0,990 & 1,966 & 1,832 & 0,297 \\
\hline & $\left(\mathrm{d}_{\mathrm{vc}}\right)$ & & & & & & - & 0,476 & 1,603 & 1,288 & 1,952 & 1,892 & 0,780 \\
\hline & $\left(\mathrm{d}_{\mathrm{cp}}\right)$ & & & & & & - & 0,829 & 1,567 & 0,943 & 1,966 & 1,801 & 0,754 \\
\hline & $\left(\mathrm{d}_{\mathrm{ft}}\right)$ & & & & & & - & 0,714 & 1,382 & 0,599 & 1,999 & 1,622 & 0,202 \\
\hline & $\left(\mathrm{D}^{2}\right)$ & & & & & & - & 5,6960 & 43,790 & 10,950 & 63,320 & 27,480 & 7,100 \\
\hline \multicolumn{14}{|l|}{ 7-Carioca } \\
\hline \multirow[t]{5}{*}{$300 \mathrm{~V}$} & $\left(d_{e}\right)$ & & & & & & & - & 1,305 & 0,895 & 1,792 & 1,088 & 0,877 \\
\hline & $\left(d_{v c}\right)$ & & & & & & & - & 1,534 & 1,025 & 1,652 & 1,428 & 0,563 \\
\hline & $\left(\mathrm{d}_{\mathrm{cp}}\right)$ & & & & & & & - & 1,232 & 0,940 & 1,614 & 1,141 & 1,081 \\
\hline & $\left(\mathrm{d}_{\mathrm{ft}}\right)$ & & & & & & & - & 1,217 & 0,928 & 1,765 & 0,913 & 0,900 \\
\hline & $\left(\mathrm{D}^{2}\right)$ & & & & & & & - & 34,300 & 10,550 & 52,620 & 13,810 & 8,110 \\
\hline \multicolumn{14}{|l|}{ 8-Ouro } \\
\hline Negro & $\left(\mathrm{d}_{\mathrm{e}}\right)$ & & & & & & & & - & 1,276 & 0,868 & 1,648 & 1,352 \\
\hline & $\left(d_{v c}\right)$ & & & & & & & & - & 1,806 & 0,965 & 2,292 & 1,590 \\
\hline & $\left(\mathrm{d}_{\mathrm{cp}}\right)$ & & & & & & & & - & 1,449 & 1,370 & 1,583 & 1,770 \\
\hline & $\left(\mathrm{d}_{\mathrm{ft}}\right)$ & & & & & & & & - & 1,082 & 0,718 & 1,662 & 1,540 \\
\hline & $\left(\mathrm{D}^{2}\right)$ & & & & & & & & - & 61,600 & 25,380 & 51,990 & 53,790 \\
\hline 9-A-285 & & & & & & & & & & & & & \\
\hline Rudá & $\left(\mathrm{d}_{\mathrm{e}}\right)$ & & & & & & & & & - & 2,063 & 1,390 & 0,844 \\
\hline & $\left(d_{\mathrm{vc}}\right)$ & & & & & & & & & - & 1,855 & 1,244 & 0,509 \\
\hline & $\left(\mathrm{d}_{\mathrm{cp}}\right)$ & & & & & & & & & - & 1,951 & 1,470 & 0,919 \\
\hline & $\left(\mathrm{d}_{\mathrm{ft}}\right)$ & & & & & & & & & - & 1,781 & 1,760 & 0,711 \\
\hline & $\left(\mathrm{D}^{2}\right)$ & & & & & & & & & - & 88,510 & 23,330 & 4,890 \\
\hline $\begin{array}{l}\text { 10-ESAL- } \\
693\end{array}$ & & & & & & & & & & & & & \\
\hline 693 & $\left(d_{e}\right)$ & & & & & & & & & & - & 2,153 & 1,886 \\
\hline & $\left(\mathrm{d}_{\mathrm{vc}}\right)$ & & & & & & & & & & - & 1,777 & 1,760 \\
\hline & $\left(\mathrm{d}_{\mathrm{cp}}\right)$ & & & & & & & & & & - & 1,641 & 1,889 \\
\hline & $\left(\mathrm{d}_{\mathrm{ft}}\right)$ & & & & & & & & & & - & 1,993 & 2,146 \\
\hline & $\left(\mathrm{D}^{2}\right)$ & & & & & & & & & & - & 54,690 & 75,120 \\
\hline 11-Pérola & $\left(d_{e}\right)$ & & & & & & & & & & & - & 1,876 \\
\hline & $\left(d_{\mathrm{vc}}\right)$ & & & & & & & & & & & - & 1,385 \\
\hline & $\left(d_{\mathrm{cp}}\right)$ & & & & & & & & & & & - & 1,764 \\
\hline & $\left(\mathrm{d}_{\mathrm{ft}}\right)$ & & & & & & & & & & & - & 1,800 \\
\hline & $\left(\mathrm{D}^{2}\right)$ & & & & & & & & & & & - & 24,840 \\
\hline 12-IAC Car & ca Aruã & & & & & & & & & & & & - \\
\hline
\end{tabular}

Ciência Rural, v. 32, n. 2, 2002. 
Tabela 2 - Coeficientes de correlação de Spearman (rs) entre estimativas de dissimilaridade de pares de genótipos de feijoeiro, obtidas pela distância Euclidiana média $\left(\mathrm{d}_{\mathrm{e}}\right)$, variáveis canônicas $\left(\mathrm{d}_{\mathrm{vc}}\right)$, componentes principais $\left(\mathrm{d}_{\mathrm{Cp}}\right)$, cargas fatoriais $\left(\mathrm{d}_{\mathrm{ft}}\right)$ e distância de generalizada de Mahalanobis $\left(\mathrm{D}^{2}\right)$, obtidas a partir de 10 caracteres agromorfológicos, avaliados na média das quatro épocas (inverno/97, águas/97/98, seca/98 e inverno/98).

\begin{tabular}{lllll}
\hline & $\mathrm{d}_{\mathrm{vc}}$ & $\mathrm{d}_{\mathrm{cp}}$ & $\mathrm{d}_{\mathrm{ft}}$ & $\mathrm{D}^{2}$ \\
\hline $\mathrm{d}_{\mathrm{e}}$ & $0,763 * *$ & $0,883^{* *}$ & $0,874 * *$ & $0,701^{* *}$ \\
$\mathrm{~d}_{\mathrm{vc}}$ & - & $0,733^{* *}$ & $0,630^{* *}$ & $0,847 * *$ \\
$\mathrm{~d}_{\mathrm{cp}}$ & & - & $0,776^{* *}$ & $0,760^{* *}$ \\
$\mathrm{~d}_{\mathrm{ft}}$ & & & - & $0,644 * *$ \\
$\mathrm{D}^{2}$ & & & & - \\
\hline
\end{tabular}

$* *=$ significativa a $1 \%$ de probabilidade pelo teste $\mathrm{t}$.

$\mathrm{Na}$ tabela 3, estão apresentadas as correlações residuais entre os dez caracteres avaliados, na qual se verifica que elas foram, em sua maioria, de baixa magnitude, ocorrendo apenas dez correlações com significância dentre as 45 observadas, quando comparadas com zero, pelo teste t. Entretanto, ao se testar a hipótese de que a matriz de correlações residuais não difere da matriz identidade $\left(\mathrm{H}_{0}: \mathrm{R}=\mathrm{I}\right)$, constatou-se que ela não deve ser rejeitada a $5 \%$ de probabilidade $\left(\chi^{2}=14,34 \mathrm{e}\right.$ g.l= 45). Apesar disso, acusou-se a presença de algumas correlações residuais significativas (Tabela 4), o que pode ter contribuído para que ocorressem diferenças entre a $D^{2}$ e $d_{\mathrm{e}}$. Esse resultado é diferente do obtido por CRUZ et al. (1994), que verificaram que a matriz de correlações residuais foi estatisticamente diferente da matriz identidade a $5 \%$ de probabilidade, por Fonseca (1997), onde os coeficientes de correlação entre os dez caracteres avaliados originais e as duas primeiras variáveis canônicas indicaram que todos os caracteres foram significativos com pelo menos uma dessas variáveis, e por Coimbra (2000), sendo que o coeficiente de correlação entre os caracteres número de dias entre a emergência e o florescimento, número de dias entre a emergência e maturação de colheita, estatura de planta e estatura de inserção do primeiro legume com o rendimento de grãos foi negativa e os caracteres número de legumes por planta e massa de mil grãos evidenciaram um grau de associação elevado com o caráter rendimento de grãos por unidade de área.

As estimativas dos autovalores $\mathrm{e}$ as variâncias associadas estão apresentadas na tabela 4 . Analisando os três primeiros componentes principais, percebe-se que a variação contida nos caracteres originais aumentou consideravelmente quando neles se incluíram a terceira raiz, principalmente para os componentes principais (Tabela 4). Para as variáveis canônicas, a primeira variável apresentou uma variação significativa, no entanto, não houve aumento considerável desta variabilidade quando nelas se incluíram a segunda e terceira raízes. Estes resultados discordam dos obtidos por CRUZ et al. (1994), que constataram que ambas as técnicas de análise por variáveis canônicas ou por componentes principais exploraram mais de $94 \%$ da variância total disponível, utilizando as duas primeiras raízes características e praticamente esgotaram esta variabilidade quando nelas se incluiu a terceira raiz, de Oliveira (1989), que verificou que os três primeiros componentes principais acumularam uma explicação da variância em $80 \%$ e que os métodos de componentes principais, análise de agrupamento e análise discriminante, apresentaram resultados similares, e de Fonseca (1997), onde as duas primeiras variáveis foram suficientes para explicar $85,5 \%$ da variação observada, sendo que a primeira variável mais importante de todas explicou $72,6 \%$ e a segunda $12,9 \%$, e não foram identificados caracteres redundantes entre os dez estudados.

Com relação à identificação dos caracteres de menor importância, na determinação da divergência genética dos genótipos, não houve concordância entre os dois métodos $\left(\mathrm{d}_{\mathrm{cp}}\right.$ e $\left.\mathrm{d}_{\mathrm{vc}}\right)$ com relação aos caracteres menos divergentes. No entanto, houve concordância em ambos os métodos apenas para um dos caracteres que, no caso, é o NLP. Constata-se, pela tabela 4, que os caracteres: NLP, NI, NR e NSL, em relação à análise por componentes principais, e PS, PD, NLP e IL, em relação a análise por variáveis canônicas, foram os que menos contribuíram para a divergência dos genitores. CRUZ et al. (1994) e FONSECA (1997) encontraram resultados satisfatórios para ambos os métodos. Os primeiros verificaram que, dos oito caracteres estudados, cinco foram os que menos contribuíram para a divergência dos genitores estudados; o segundo observou que todos os dez caracteres avaliados foram significativos com, pelo menos, uma das variáveis estudadas.

Com base nos componentes principais, os caracteres NLP e NI, possivelmente, tiveram menor importância para a diversidade entre os genótipos, por terem apresentado o menor quociente entre os 
Tabela 3 - Estimativas das correlações residuais entre dez caracteres avaliados na média das quatro épocas (inverno/97, águas/97/98, seca/98 e inverno/98) em doze cultivares/linhagens de feijão.

\begin{tabular}{|c|c|c|c|c|c|c|c|c|c|c|}
\hline Caráter $^{1}$ & $\mathrm{FL}^{\underline{1 /}}$ & $\mathrm{NLP}^{1 /}$ & $\mathrm{NI}^{\underline{1 /}}$ & $\mathrm{PS}^{1 /}$ & $\mathrm{PD}^{1 /, 2 l}$ & $\mathrm{NLV}^{\underline{1 /}}$ & $\mathrm{IL}^{\underline{1 /}}$ & $\mathrm{NR}^{\underline{1 /}}$ & $\mathrm{HP}^{1 /, 3 /}$ & $\mathrm{PR}^{\underline{1}}$ \\
\hline FL & 1 & $-0,176$ & $-0,177$ & $-0,030$ & $-0,244$ & $-0,316$ & $-0,199$ & $-0,102$ & $-0,218$ & $-0,118$ \\
\hline NVP & & 1 & $0,230^{*}$ & $-0,120$ & $0,226 *$ & 0,165 & $-0,072$ & $0,377 * *$ & 0,186 & 0,146 \\
\hline NI & & & 1 & $-0,146$ & 0,149 & 0,018 & $-0,052$ & $0,580 * *$ & $0,707 * *$ & 0,190 \\
\hline PS & & & & 1 & $-0,009$ & $-0,023$ & 0,079 & $-0,088$ & 0,028 & 0,045 \\
\hline PD & & & & & 1 & $0,216^{*}$ & 0,109 & $0,316^{* *}$ & 0,187 & 0,100 \\
\hline NSV & & & & & & 1 & 0,022 & 0,155 & 0,049 & 0,022 \\
\hline IV & & & & & & & 1 & $-0,288$ & $0,315^{* *}$ & 0,118 \\
\hline NR & & & & & & & & 1 & $0,317 * *$ & 0,154 \\
\hline HP & & & & & & & & & 1 & $0,300 * *$ \\
\hline PR & & & & & & & & & & 1 \\
\hline
\end{tabular}

1/: FL: número de dias de florescimento; NLP: número médio de legumes/planta; NI: número médio de internódios da haste principal; PS: peso médio de 100 sementes (gramas); PD: produção de grãos em kg/ha; NLV: número médio de sementes/legume; IL: inserção média do primeiro legume $(\mathrm{cm})$; NR: número médio de ramos por planta; HP: comprimento médio da haste principal (cm) e PR: porte das plantas (nota) ${ }^{2 /:} 10^{-5} ; \mathrm{e}^{3 /:} 10^{-2} * \mathrm{e}^{* *}=$ significativas a 5 e $1 \%$ de probabilidade pelo teste $\mathrm{t}$, respectivamente.

Tabela 4 - Estimativas das variâncias (autovalores, $\mathrm{U}_{\mathrm{i}}$ ), associadas aos componentes principais, às variáveis canônicas, respectivos coeficientes de ponderação (autovetores) e ao QMR de dez caracteres avaliados na média das quatro épocas (inverno/97, águas/97/98, seca/98 e inverno/98).

\begin{tabular}{|c|c|c|c|c|c|c|c|c|c|c|c|}
\hline $\mathrm{U}_{\mathrm{i}}$ & $\begin{array}{c}\text { Variação } \\
\text { Acumulada } \\
(\%)\end{array}$ & $\mathrm{FL}^{\stackrel{1 /}{-}}$ & $\mathrm{NLP}^{1 /}$ & $\mathrm{NI}^{1 /}$ & $\mathrm{PS}^{\underline{1} /}$ & $\mathrm{PD}^{1 /, \underline{l}}$ & $\mathrm{NSL}^{\underline{1}}$ & $\mathrm{IL}^{\underline{1}}$ & $\mathrm{NR}^{1 /}$ & $\mathrm{HP}^{1} \underline{1,}, \underline{3}$ & $\mathrm{PR}^{1 /}$ \\
\hline & & & & & Componentes & Principais & & & & & \\
\hline 3,926 & 39,256 & 0,283 & 0,088 & 0,453 & $-0,484$ & $-0,247$ & 0,099 & $-0,449$ & $-0,134$ & $-0,288$ & $-0,414$ \\
\hline 2,332 & 62,573 & 0,414 & $-0,188$ & $-0,012$ & 0,240 & $-0,518$ & 0,337 & $-0,223$ & 0,150 & 0,265 & 0,459 \\
\hline 1,729 & 79,859 & 0,474 & $-0,121$ & 0,106 & $-0,036$ & 0,217 & 0,032 & 0,443 & $-0,507$ & $-0,352$ & 0,349 \\
\hline 1,082 & 90,680 & $-0,449$ & $-0,268$ & $-0,006$ & 0,048 & $-0,173$ & $-0,110$ & $-0,207$ & 0,127 & $-0,679$ & 0,404 \\
\hline 0,558 & 96,266 & $-0,074$ & 0,226 & 0,431 & 0,642 & $-0,383$ & $-0,066$ & 0,273 & $-0,095$ & $-0,163$ & $-0,290$ \\
\hline 0,196 & 98,224 & 0,224 & 0,512 & 0,044 & 0,204 & 0,435 & 0,331 & $-0,124$ & 0,472 & $-0,307$ & 0,114 \\
\hline 0,085 & 99,073 & $-0,396$ & $-0,012$ & 0,304 & $-0,334$ & $-0,084$ & 0,638 & 0,440 & 0,104 & 0,099 & 0,094 \\
\hline 0,048 & 99,555 & 0,309 & $-0,471$ & $-0,201$ & $-0,053$ & $-0,103$ & 0,025 & 0,340 & 0,506 & $-0,264$ & $-0,431$ \\
\hline 0,030 & 99,857 & 0,065 & $-0,246$ & 0,663 & $-0,036$ & 0,255 & $-0,424$ & 0,037 & 0,366 & 0,245 & 0,233 \\
\hline \multirow[t]{2}{*}{0,014} & 100,000 & $-0,095$ & $-0,523$ & 0,151 & 0,367 & 0,418 & 0,402 & $-0,329$ & $-0,233$ & 0,034 & $-0,244$ \\
\hline & & & & & Variáveis & Canônicas & & & & & \\
\hline 5055,502 & 99,770 & $-0,013$ & $-0,370$ & 0,706 & $-0,319$ & 0,041 & 0,036 & $-0,210$ & $-0,297$ & $-0,358$ & $-0,001$ \\
\hline 7,574 & 99,920 & $-0,003$ & $-0,151$ & 0,167 & 0,079 & $-0,323$ & 0,343 & $-0,135$ & $-0,334$ & 0,769 & $-0,002$ \\
\hline 1,930 & 99,958 & $-0,002$ & $-0,365$ & 0,305 & 0,334 & $-0,200$ & 0,157 & 0,031 & 0,772 & 0,014 & 0,007 \\
\hline 1,090 & 99,980 & 0,005 & 0,723 & 0,366 & $-0,133$ & $-0,112$ & $-0,121$ & $-0,462$ & 0,267 & 0,118 & 0,001 \\
\hline 0,509 & 99,990 & $-0,705$ & $-0,020$ & $-0,024$ & 0,004 & 0,014 & $-0,036$ & $-0,046$ & 0,003 & 0,016 & 0,705 \\
\hline 0,275 & 99,995 & 0,707 & $-0,007$ & 0,009 & $-0,005$ & 0,014 & $-0,003$ & 0,001 & $-0,005$ & 0,007 & 0,707 \\
\hline 0,098 & 99,997 & $-0,034$ & 0,204 & 0,365 & $-0,248$ & $-0,103$ & $-0,192$ & 0,823 & 0,035 & 0,189 & 0,027 \\
\hline 0,076 & 99,998 & $-0,030$ & 0,332 & $-0,009$ & 0,086 & $-0,281$ & 0,763 & 0,187 & $-0,106$ & $-0,414$ & 0,045 \\
\hline 0,012 & 99,998 & $-0,012$ & 0,153 & 0,281 & 0,418 & 0,798 & 0,231 & 0,086 & $-0,066$ & 0,144 & $-0,003$ \\
\hline 0,072 & 100,000 & 0,005 & 0,097 & 0,184 & 0,721 & $-0,339$ & $-0,408$ & 0,033 & $-0,340$ & $-0,197$ & 0,003 \\
\hline QMR & & 1,483 & 7,632 & 1,335 & 7,652 & 4,364 & 0,280 & 1,335 & 3,658 & 1,256 & 0,217 \\
\hline
\end{tabular}

11: FL: número de dias de florescimento; NLP: número médio de legumes/planta; NI: número médio de internódios da haste principal; PS: peso médio de 100 sementes (gramas); PD: produção de grãos em kg/ha; NSL: número médio de sementes/legume; IL: inserção média do primeiro legume (cm); NR: número médio de ramos por planta: HP: comprimento médio da haste principal $(\mathrm{cm})$ e PR: porte das plantas (nota): ${ }^{2 !}: 10^{-5} \mathrm{e}^{\underline{3 l}:} 10^{-2}$.

quadrados médios de tratamentos e de resíduos (dados não apresentados). Os caracteres NLP e NR foram também considerados de menor importância para as variáveis canônicas, possivelmente em razão do aspecto de redundância.

A concordância parcial entre os cinco métodos multivariados empregados pode ser visualizada ao se analisarem os correspondentes dendogramas, oriundos das análises de agrupamentos (Figura 1). Assumindo como ponto de corte nos dendogramas a metade da amplitude das distâncias, obtém-se sete grupos, considerando-se tanto a $\mathrm{d}_{\mathrm{e}}$ (Figura 1a), quanto a $\mathrm{d}_{\mathrm{ft}}$ (Figura 1b). Com base na $d_{c p}$ (Figura 1c) ou da $d_{v c}$ (Figura 1d), formaram-se cinco grupos considerando-se a $\mathrm{D}^{2}$, apenas três grupos (Figura 1e). Em quatro dos cinco procedimentos, os genótipos ESAL 693 e Ouro Negro isolaram-se das demais, como era esperado, 


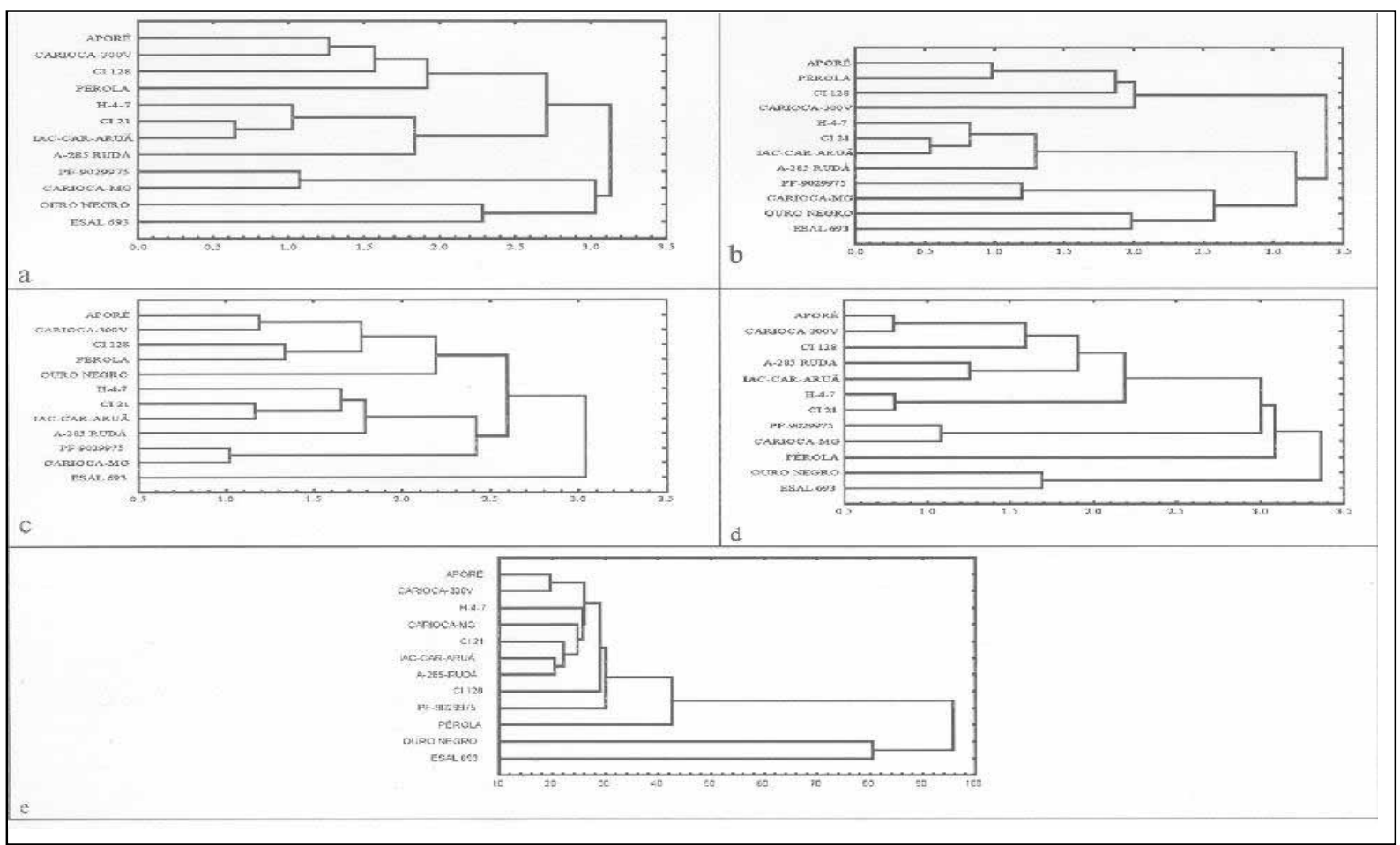

Figura 1 - Dendogramas resultantes da análise de agrupamento utilizando o Método UPGMA a partir da média das quatro épocas (inverno/97, águas/97/98, seca/98 e inverno/98) com base nas distâncias Euclidianas médias com dados originais padronizados (a); nas cargas fatoriais (b), nos componentes principais (c), variáveis canônicas (d) e na distância generalizada de Mahalanobis $\mathrm{D}^{2}$ (e).

uma vez que o primeiro é oriundo do cruzamento entre Carioca TU x Linha 3272, e o segundo é uma introdução de Honduras e possui grão preto, portanto, possuem origens e características diferente dos demais. Assim, o cruzamento desses genótipos, entre si ou com os demais, devem produzir as populações mais segregantes. Como na maioria das regiões brasileiras a preferência é por cultivares com grãos tipo carioca, são promissores os cruzamentos envolvendo o genótipo ESAL 693 com os demais. Qualquer das $d_{e}$ isolaram o PF-9029975 e Carioca MG dos demais. Também por meio das $d_{e}$, nota-se a divergência entre os genótipos CI-128 e CI-21, os quais são ambos provenientes do ciclo I de um programa de seleção recorrente. A divergência entre eles mostra a ampla variabilidade na população segregante do programa em apreço e sugere a possibilidade de seleção de novas linhagens superiores. Chama a atenção, pelo menos em dois procedimentos de $d_{e}$, a divergência entre os genótipos Aporé e Pérola, uma vez que o Pérola foi selecionado a partir do Aporé.

\section{CONCLUSÕES}

O genótio ESAL 693 é o genitor mais promissor para se obter populações com ampla variabilidade genética e grãos tipo carioca, por ser o mais divergente.

As técnicas multivariadas foram parcialmente concordantes na identificação da divergência genética entre os genótipos.

\section{AGRADECIMENTOS}

Trabalho financiado com o auxílio financeiro do $\mathrm{CNPq} / \mathrm{FAPEMIG}$

\section{REFERÊNCIAS BIBLIOGRÁFICAS}

ABREU, A. de. F.B., RAMALHO, M.A.P., FERREIRA, D.F Selection potential for seed yield from intra - end inter - racial populations in common bean. Euphytica, Wageningen, v.108, n.2, p.121-127, 1999 .

ADAMS, M.W. An estimation of homogeneity in crop plants, with special reference to genetic vulnerability in the dry bean, Phaseolus vulgaris L. Euphytica, Wageningen, v.26, 665679, 1977

COIMBRA, J.L.M., GUIDOLIN, A.F, CARVALHO, F.I.F.de., et al. Correlacões canônicas: II - Análise do rendimento de grãos de feijão e seus componentes. Ciência Rural, Santa Maria, v.30, n.1, p.31-35, 2000.

CRUZ, C.D. Aplicação de algumas técnicas multivariadas no melhoramento de plantas. Piracicaba, 1990. 188 p. Tese (Doutorado em Genética e Melhoramento de Plantas) ESALQ/USP, 1990 
CRUZ, C.D. Aplicativo computacional em genética e estatística. Viçosa : UFV, 1997. 442p.

CRUZ, C.D., CARVALHO, S.P. de, VENCOVSKY, R. Estudos sobre divergência genética. II Eficiência da predição do comportamento de híbridos com base na divergência de progenitores. Revista Ceres, Viçosa, v.41, n.234, p.183-190, 1994

CRUZ, C.D., REGAZZI, A.J. Modelos biométricos aplicados ao melhoramento genético. Viçosa : UFV, 1997. 390p.

CUARTERO, J.; GOMES-GUILLAMON, M.L.; DIAZ, G. $\boldsymbol{e}$ t. al Agropacion intra-especifica en variedades de pimento. Anales de Edafologia y Agrobiologia, v.42, n.718, p.1209$1219,1983$.

DIAS, L.A. Análises multidimensionais. In: ALFENAS, A.C.(ed.) Eletroforese de isoenzimas e proteínas afins. Viçosa : UFV, 1998. p.405-475.

FARIAS, J.M., THOMAS, N., QUIROGAG, H.M. Utilizacion del análisis de componentes principales en la selection de lineas y variedades introducidas de ballico anual, Lolium multiflorum Lam. Agric Téc Méx, v.9, n.2, p.125-140, 1983.

FONSECA, J.R., SILVA, H.T. da. Emprego da análise multivariada na carcaterização de acessos de feijão (Phaseolus vulgaris L.). Revista Brasileira de Sementes, v. 19, n.2, p.335-341, 1997.

FERREIRA, D.F. Métodos de avaliação da divergência genética em milho e suas relações com os cruzamentos dialélicos. Lavras, 1993. 72p. Dissertação (Mestrado em Genética e Melhoramento de Plantas) - Lavras-UFLA, 1993.

GHADERI, A., ADAMS, M.W., NASSIB, A.M. Relationship between genetic distance and heterosis for yield and morphological traits in dry edible bean and faba bean. Crop Science, Madison. v.14, n.1, p.24-27, 1984.

GODOI, C.R. de M. Análise estatística multidimensional Piracicaba : ESALQ/USP, 1985. 187p.

HUSSAINI, S.H., GOODMAN, M.M, TIMOTHY, D.H Multivariate analysis and the geographical distribuition of the collection of finger millet. Crop Science, Madison, v.17, n.2, p. $257-263,1977$

ISEBRANDS, J.G., CROW, T.R. Introduction to uses and interpretation of principal component analysis in forest biology. Minnesota, US : Department of Agriculture, 1975. $18 \mathrm{p}$

JAIN, A.K., BHAGAT, N.K., TIWART, A.S. Genetic divergence inf finger millet. Indian Journal of Genetics and Plant Breeding, New Delhi v.41, p.346-348, 1981a.

JAIN, K.C., PANDYA, B.P., PANDE, K. Genetic divergence in chickpea. Indian Journal of Genetics and Plant Breeding, New Delhi, v.41, p.220-225, 1981b
JOHNSON, R.A., WICHERN, D.W. Applied multivariate statistical analysis. Englewood Cliffs, New Jersey : PrenticeHall, 1992. 642p

JULQUIFAR, A.W., VIRMANI, S.S., CARPENA, A.L. Genetic divergence among some maintainer and restorer lines in relation to hybrid breeding rice (Oryza sativa L.) Theoretical Applied Genetics, Berlin, v.70, p.671-678, 1985 .

MACHADO, C.F., SANTOS, J.B. dos., NUNES, G.H.de S Escolha de genitores de feijoeiro por meio da divergência baseada em caracteres morfo-agronômicos. Bragantia, Campinas, v.59, n.1, 11-20, 2000

MACHADO, C.F., SANTOS, J.B. dos., NUNES, G.H.de S. Choice of common bean parents based on combining ability estimates. Brazilian Journal of Genetics, Ribeirão Preto, 2002. (Prelo)

MALUF, W.R., FERREIRA, P.E., MIRANDA, J.E.C. Genetic divergence in tomatoes and its relationship with heterosis for yield in $F_{1}$ hybrids. Revista Brasileira de Genética, Ribeirão Preto. V.3, p.453-460, 1983.

MARDIA, K.V., KENT, J.T., BIBBY, J.M. Multivariate analisis. London : Academic, 1979. 521p.

MIRANDA, J.E.C., CRUZ, C.D., COSTA, C.P. Predição do comportamento de híbridos de pimentão (Capsicum annum L.) pela divergência genética dos progenitores. Revista Brasileira de Genética, Ribeirão Preto, v.11, p.929-937, 1988.

MIRANDA, G.V. Diversidade genética e desempenho de cultivares de soja como progenitores. Viçosa, 1998. 117p Tese (Doutorado em Genética e Melhoramento de Plantas) Viçosa-UFV, 1998

OLIVEIRA, E.J. de. Análise multivariada no estudo da divergência genética entre cultivares de feijão (Phaseolus vulgaris L.). Viçosa:UFV, $1989 . \quad 91 p . \quad$ Dissertação (Mestrado em Genética e Melhoramento de Plantas)- ViçosaUFV, 1989.

OLIVEIRA, L.B., RAMALHO, M.A.P, ABREU. $\boldsymbol{e}$ t al. Alternative procedures for parent choice in a breeding program for the common bean (Phaseolus vulgaris L.). Brazilian Journal of Genetics, Ribeirão Preto, v.19, n.4 p.611-615, 1996.

TATSUOKA, M.M. Multivariate analysis. New York : Wiley, 1971. 451p.

VIEIRA, C. Influência das épocas de plantio sobre as etapas de desenvolvimento do feijoeiro (Phaseolus vulgaris L.). Revista Ceres, Viçosa, v.38, n.219, p.438-443, 1991.

VIEIRA, C., ARAÚJO, G.A.de A., CARDOSO, A.A. Triagem de germoplasma de feijão (Phaseolus vulgaris L.) em busca de fontes de tolerância à baixa temperatura - II. Revista Ceres, Viçosa, v.47, n.271, p.337-348, 2000. 